\title{
Arborescences
}

Revue d'études françaises

\section{La dynamique de la circulation des savoirs et de leurs matériaux dans les échanges épistolaires entre Jean-Baptiste Boisot et Paul Pellisson-Fontanier (1674-1693)}

\section{Corinne Marchal}

Numéro 9, décembre 2019

La lettre érudite. Nouvelles recherches sur la communication savante à l'époque moderne (XVI ${ }^{\mathrm{e}}$-XVIII ${ }^{\mathrm{e}}$ siècles)

URI : https://id.erudit.org/iderudit/1068277ar

DOI : https://doi.org/10.7202/1068277ar

Aller au sommaire du numéro

Éditeur(s)

Département d'études françaises, Université de Toronto

ISSN

1925-5357 (numérique)

Découvrir la revue

Citer cet article

Marchal, C. (2019). La dynamique de la circulation des savoirs et de leurs matériaux dans les échanges épistolaires entre Jean-Baptiste Boisot et Paul Pellisson-Fontanier (1674-1693). Arborescences, (9), 95-105.

https://doi.org/10.7202/1068277ar
Résumé de l'article

123 lettres sont conservées de la correspondance (1674-1693) entre Jean-Baptiste Boisot, érudit comtois héritier des archives et de la collection des Granvelle, et Paul Pellisson-Fontanier, l'un des plus brillants hommes de lettres de son temps, controversiste et historiographe du roi Louis XIV. Cette correspondance se présente sous la forme d'échanges suivis, réguliers, marqués par une circulation abondante et dynamique de savoirs et d'objets de connaissance. L'inégalité de leur situation, sur un plan social autant que scientifique, laissa néanmoins à Jean-Baptiste Boisot l'opportunité de tirer profit de la bienveillante protection de Pellisson pour diffuser les trésors de sa collection et se faire connaître comme un érudit aux projets originaux et audacieux, plutôt que comme un servile collaborateur du controversiste. Cette étude a pour propos d'éclairer les avantages qu'un docte d'une province périphérique réunie depuis peu à la France pouvait tirer, quant à la circulation des savoirs, de ses échanges épistolaires avec un savant de renom et bien en cour, au fil d'une relation en constant renouvellement.
Tous droits réservés (c) Département d'études françaises, Université de Toronto, 2020 cotégé par la loi sur le droit d'auteur. L'utilisation des services d'Érudit (y compris la reproduction) est assujettie à sa politique d'utilisation que vous pouvez consulter en ligne. 


\section{SOMMAIRE}

1 Sébastien Drouin, Université de Toronto

Camelia Sararu, Université de Toronto

Introduction

10 Myron McShane, Centre d'études sur la Réforme et la Renaissance, Université de Toronto Une lettre de Jean Dorat sur l'œuvre de Nonnos

31 Benoît Autiquet, Université de Bâle

Ce que la lettre familière fait au discours médical.

Une lecture de la lettre XIX, 16 des Lettres de Pasquier (1619)

49 Guillaume Bazière, Université Paris Nanterre

Présence érudite et savoirs politiques dans la correspondance du Grand Condé

68 Vanezia Pârlea, Université de Bucarest

Lettres d'Orient: échanges épistolaires en contexte interculturel dans les Mémoires du chevalier d'Arvieux

81 Yves Moreau, Université de Lyon

"Qualche novità litteraria»: la correspondance

entre Jacob Spon (1647-1685) et Antonio Magliabechi (1633-1714)

95 Corinne Marchal, Université de Franche-Comté

La dynamique de la circulation des savoirs et de leurs matériaux dans les échanges épistolaires entre Jean-Baptiste Boisot et Paul Pellisson-Fontanier (1674-1693)

106 Mathilde Chollet, Le Mans Université

Un «gai savoir»: stratégies du rire dans les lettres

$d$ 'une érudite des Lumières 


\title{
La dynamique de la circulation des savoirs et de leurs matériaux dans les échanges épistolaires entre Jean-Baptiste Boisot et Paul Pellisson-Fontanier (1674-1693)
}

\author{
Corinne Marchal, Université de Franche-Comté
}

Résumé

123 lettres sont conservées de la correspondance (1674-1693) entre Jean-Baptiste Boisot, érudit comtois héritier des archives et de la collection des Granvelle, et Paul PellissonFontanier, l'un des plus brillants hommes de lettres de son temps, controversiste et historiographe du roi Louis XIV. Cette correspondance se présente sous la forme d'échanges suivis, réguliers, marqués par une circulation abondante et dynamique de savoirs et d'objets de connaissance. L'inégalité de leur situation, sur un plan social autant que scientifique, laissa néanmoins à Jean-Baptiste Boisot l'opportunité de tirer profit de la bienveillante protection de Pellisson pour diffuser les trésors de sa collection et se faire connaître comme un érudit aux projets originaux et audacieux, plutôt que comme un servile collaborateur du controversiste. Cette étude a pour propos d'éclairer les avantages qu'un docte d'une province périphérique réunie depuis peu à la France pouvait tirer, quant à la circulation des savoirs, de ses échanges épistolaires avec un savant de renom et bien en cour, au fil d'une relation en constant renouvellement.

Dans cet «interrègne de la pensée française» que fut la période entre les grands classiques et les philosophes des Lumières, où triomphaient les amateurs de curiosités scientifiques et les maîtres de la polémique religieuse, où les traducteurs et restituteurs de textes gouvernaient (Goncourt 1903: 75-76), le Bisontin Jean-Baptiste Boisot (1639-1694 ${ }^{1}$ ) se fit connaître de la République des Lettres par sa collection, principalement formée de celle des Granvelle et de leurs archives. Il devait acquérir auprès des doctes la réputation d'un "grand babioliste» et d'un "bijoutier", surnoms qu'il se donnait lui-même, se faisant auprès d'eux le dispensateur généreux de "présents sacrés et profanes».

S’il fut un prolifique épistolier qui «recevoit de France, d'Allemagne, d'Italie, d'Espagne \& de presque tous les endroits de l'Europe des lettres de la plupart des personnes les plus distinguées par leur mérite \& par leur sçavoir» (Bosquillon, «Lettre» : 393), il ne demeure que 229 missives actives et surtout passives de cette correspondance. À l'image de bien des contemporains, Boisot demeura sélectif quant à leur conservation. Il veilla à archiver 120 lettres non autographes reçues de Paul PellissonFontanier entre le 16 août 1674 et le 27 janvier $1693^{2}$. Si cet illustre correspondant lui confia un jour qu'il conservait, "par estime et par amitié», toutes celles que lui envoyait le Bisontin (Pellisson, Lettres inédites: 126), trois lettres seulement nous sont parvenues (Pellisson, Additions: 69-76; 94-95).

Durant toute cette période, Pellisson se trouva "partout où était le roi» (Marcou 1859: 278), à son lever et à son coucher, à Saint-Germain comme à Versailles, dans les Flandres ou en Hollande, en

1. Ses biographes le font naître à tort en 1638. Il fut baptisé à l'église Saint-Pierre le 8 mai 1639 (Registres paroissiaux, GG 177: fo $\left.30 \mathrm{r}^{\circ}\right)$.

2. Voir Pellisson-Fontanier, Lettres [...] à M. Boisot (ms 602). 
Franche-Comté ou sur les bords du Rhin. Il fut son historiographe de 1670 à 1677 et devint en 1676 l'administrateur de la caisse des Économats, que les protestants appelaient par dérision la «caisse des Conversions», parce qu'elle procurait des secours à leurs anciens coreligionnaires qui s'étaient convertis au catholicisme. Depuis qu'il avait solennellement abjuré le calvinisme le 8 octobre 1670, l'homme de lettres et académicien avait placé sa plume au service d'un prosélytisme qui n'avait pas vocation de contraindre mais de convaincre. La révocation de l'Édit de Nantes n'altéra pas cet irénisme, qui trouva son expression à partir de 1686 dans des ouvrages de controverse répondant aux contradicteurs d'Angleterre, de Hollande et d'Allemagne.

La correspondance entre Jean-Baptiste Boisot et Paul Pellisson-Fontanier, ces « deux doctes entre Dieu et le roi» (Marchal 1996), se présente sous la forme d'échanges suivis, marqués par une circulation abondante et dynamique de savoirs et de leurs objets de connaissance (Isaac et Sorgeloos 1996), échanges dont les contenus furent à l’origine très variés. La spécialisation s'opéra à mesure que se formait une collaboration entre les deux érudits. Il conviendra de déterminer l'effort de chacun à contrôler cette circulation érudite, à l'enrichir et à la faire évoluer, en considérant l'inégalité de leurs rapports, lesquels furent longtemps clientélistes et régis par des statuts très différents au sein de la République des Lettres.

\section{Envois variés et intéressés du collectionneur au commensal du roi}

\subsection{Objets de curiosité et mémoires savants pour plaire au roi}

Le premier témoignage de cette correspondance porte sur les rapports intimes que les deux épistoliers entretenaient avec la Cour. Dès que la Comté eut été promise à la France par la conquête de 1674, Boisot chercha à tirer avantage de la position fort influente de Pellisson auprès de Louis XIV. Le Comtois avait probablement fait la connaissance de l'homme de lettres lors d'un long séjour d'études à Paris, vers 1658-1659, mais la première missive conservée ne date que du 16 août 1674, trois mois après la capitulation accordée à la ville de Besançon. À la demande du Bisontin, Pellisson y joignit une recommandation pour le gouverneur de Franche-Comté ainsi qu'une autre pour l'intendant. Dans sa quête d'un bénéfice ecclésiastique plus enviable que ceux dont il jouissait, Boisot cherchait à se faire connaître des deux plus hautes autorités que le pouvoir central avait placées depuis peu dans la province conquise. Mais il ne se limita pas à cette démarche et s'appuya surtout sur Pellisson pour être remarqué du roi ou de son confesseur, le père La Chaise, lequel était détenteur de la feuille des bénéfices, leur adressant indirectement, par voie épistolaire, une grande variété de présents érudits et curieux. Ceux-ci circulèrent jusqu'en 1686 dans le cadre officiel d'un système de fidélité et de clientèle, où Pellisson joua avec bienveillance et avec zèle le rôle d'intercesseur.

La Cour offrait des ressources pour la recherche; elle était un lieu de concentration d'objets de curiosité et d'étude qui s'enrichissait régulièrement de dons et de nouvelles acquisitions, ainsi qu'un terrain d'expérimentations pour les savants (Saule et Arminjon 2010). Boisot désira contribuer au développement de ces collections, ce qui était un moyen de participer au progrès scientifique tout en plaisant au roi. Louis XIV avait une inclination pour les médailles. L'on prétendait qu'elle lui venait de François de La Chaise, médailliste réputé, mais ce goût s'était révélé plus tôt chez lui. Depuis 1664, la collection royale ne cessait de s'enrichir de pièces rares et singulières par l'achat sélectif ou en totalité de cabinets d'amateurs réputés (Veillon 1997). Au début de l'été 1676, Boisot fit parvenir à Pellisson l'inventaire du médaillier des Chifflet, une lignée d'érudits comtois, lequel médallier était à vendre. Le document fut aussitôt remis au père La Chaise (Pellisson, Lettres inédites: 42-43). 
En 1678, Louis XIV commanda l'aménagement du «petit appartement» de Versailles, où seraient montrées les collections royales, l'une des pièces devant accueillir les «coquilles», c'est-à-dire des fossiles de mollusques. Ces naturalia étaient très prisés des amateurs de cabinets de curiosités qui fleurissaient chez les princes, chez les particuliers éclairés ou dans certaines institutions (Mauriès 2011; Schnapper 2012). À la fin de l'été 1678, Jean-Baptiste Boisot se rendit à Fontainebleau. Ce fut dans cette résidence automnale de la Cour qu'un jour, en présence de Pellisson, il entretint un ami de son protecteur - probablement le père La Chaise - des «coquilles» de sa collection. L'hiver suivant, Pellisson le pressa d'honorer sa promesse d'envoyer ces curiosités à cet ami, qui était fort impatient de les découvrir. Pellisson se proposait non seulement de transmettre le colis à l'intéressé, mais également d'en régler le port. Il reçut ces «belles et extraordinaires coquilles» en avril 1679 à Saint-Germain-enLaye, où résidait alors la Cour (Pellisson, Lettres inédites: 47).

Les curiosités présentées par Boisot au monarque ou à son confesseur ne furent pas toutes accueillies avec autant d'intérêt. Le 11 octobre 1684, son protecteur l'informait du refus par Louis XIV d'un insolite cure-dent qui avait sans doute appartenu à Charles Quint, habitué à utiliser ce genre d'instruments en or (Ribier, Lettres et mémoires II : 598-599). C'était probablement le même objet qui était exposé au début du XVIII ${ }^{\mathrm{e}}$ siècle dans le cabinet de Claude Boisot, frère de l'Abbé alors décédé (Martène et Durand, Voyage littéraire I: 166).

Les envois du docte bisontin ne se bornèrent pas à quelques curiosités susceptibles de rejoindre les collections royales. Il tenait à prouver sa maîtrise d'une culture savante que le roi et son confesseur savaient apprécier chez un postulant à un bénéfice ecclésiastique, en un temps où maintes communautés religieuses s'efforçaient de restaurer ou de mettre en valeur les études monastiques. À l'automne 1678, Boisot adressa à son protecteur un mémoire touchant l'institution par l'empereur Auguste de l'impôt du vingtième sur les successions, «renouvelé», c'est-à-dire amendé par ses successeurs. Celui-ci avait été instauré pour atténuer le déficit du Trésor impérial lié aux dépenses des guerres civiles. L'opuscule fut montré au roi au printemps suivant (Pellisson, Lettres inédites: 46-48). Louis XIV parut intéressé par le sujet et confia au courtisan le soin d'en informer le chancelier Michel Le Tellier. La monarchie recherchait alors des expédients pour financer la coûteuse guerre de Hollande (1672-1679), et cette solution trouvée à une crise financière par un empereur qui faisait l'admiration des hommes du Grand Siècle était susceptible d'imitation.

Si Pellisson mit beaucoup de conviction et d'énergie à exercer le rôle d'intercesseur lorsqu'il s'agissait de révéler en son protégé le docte et le polymathe, il sanctionna les tentatives que fit celui-ci pour s'approprier cette culture mondaine et thuriféraire qui triomphait à la Cour, en cherchant à lui en montrer la superficialité. Un sonnet en vers bègues que Boisot avait composé au printemps 1677 en l'honneur de Mademoiselle de Bauffremont, une Comtoise habituée de la Cour, fut reçu avec froideur par le courtisan, qui n'avait pourtant pas méprisé cette poésie lorsqu'il s'était agi de flatter le souverain: "Il est fort bon en son genre, mais comme selon moi ce genre n'est guère bon, je ne vous conseillerais guère de vous y amuser, pouvant tourner de tant d'autres côtés plus agréables ou plus solides la facilité et la beauté de votre esprit» (Pellisson, Lettres inédites: 43-44). L'éloge funèbre de Marie-Thérèse d'Autriche, écrit par Boisot après la mort de cette reine, survenue le 30 juillet 1683, ne fut pas mieux considéré, et Pellisson se retint de le remettre au roi: "Je n'ai pas eu occasion et n'ai pas cru nécessaire de lui donner [...] ce que vous m'avez fait l'honneur de m'écrire sur la mort de la reine, quoiqu'il fût fort bien dit et fort bien pensé» (Pellisson, Lettres inédites: 57). 


\section{2 Éclairer I'historiographe du roi}

Si la variété des sujets caractérisa pendant dix années cette circulation de savoirs, d'objets de curiosité et d'essais littéraires ou poétiques destinés au roi et à la Cour, ceux que Boisot réservait à Pellisson furent marqués en revanche par plus d'unité, car son intention était d'éclairer son correspondant sur les particularités de la Comté et sur les acteurs de la première conquête de la province par les armées de Louis XIV. Cette transmission de connaissances était susceptible de contenter l'auteur d'une Histoire de la conquête de la Franche-Comté, laquelle ne devait être rendue publique qu'à titre posthume en 1729.

À une date indéterminée, l'érudit bisontin lui adressa un mémoire sur Jean de Watteville (16181702) (Pellisson, Lettres inédites: 81), une personnalité marquante de cette courte guerre: en 1667, alors que les Comtois s'alarmaient du bellicisme croissant de la France à leur encontre, ce gentilhomme fut chargé par les États de la province de conclure une ligue défensive avec les cantons confédérés. Il devait toutefois bientôt trahir son camp en se ralliant à la France. Pellisson consacre plusieurs pages, dans sa relation, au portrait ainsi qu'à l'action de ce personnage au cours de cette guerre (Pellisson, Histoire: 178-185). Il est possible qu'elles aient emprunté au mémoire du Bisontin, qui n’a pas été conservé.

À l'automne 1683, Boisot fit parvenir à son ami une courte relation sur saint Claude, supérieur du monastère jurassien de Saint-Oyend-de-Joux - plus tard abbaye de Saint-Claude. Cet abbé mourut au début du $\mathrm{VIII}^{\mathrm{e}}$ siècle en odeur de sainteté, mais son culte ne se répandit qu'après la découverte de son corps, vraisemblablement dans le premier quart du $\mathrm{XI}^{\mathrm{e}}$ siècle, dans un surprenant état de conservation. «Je crois voir avec vous, et n'admire pas moins que vous, ce que vous marquez du corps de saint Claude dont je n'avais pas encore entendu parler» (Pellisson, Lettres inédites: 59). Cet aveu d'ignorance de Pellisson, dans sa lettre du 20 octobre 1683, a de quoi étonner, car le sanctuaire jurassien était devenu à la fin du Moyen Âge un important lieu de pèlerinage, honoré à deux reprises par Louis $\mathrm{XI}$ ainsi que par plusieurs ducs de Bourgogne. Un climat de ferveur l'entoura de nouveau au XVII ${ }^{\mathrm{e}}$ siècle. François de Sales vint par exemple se recueillir à trois reprises auprès du corps du thaumaturge. Pellisson ne se servit pas de ce mémoire pour son œuvre et ne semble pas l'avoir diffusé autour de lui, pas plus qu'il ne s’intéressa à deux lettres que Boisot publia dans le Journal des savants du 22 juillet et du 9 septembre 1686 sur les particularités géologiques de la province, lesquelles auraient pu enrichir pourtant son tableau des singularités de la Franche-Comté, qui introduisait son récit de la conquête de 1668. Il jugea même avec sévérité le contenu de la seconde, qui décrivait la glacière naturelle de Chaux-lès-Passavant, à l'est de Besançon, affirmant qu'il en avait écrit davantage sur ce sujet dans son manuscrit, en empruntant aux Mémoires historiques de la république séquanaise publiés en 1592 par Louis Gollut (1535-1595), titulaire de la chaire de littérature latine de l'université de Dole (Pellisson, Lettres inédites: 83-84).

De 1674 à 1686, cette circulation savante par voie épistolaire entre les deux doctes fut donc uniquement entretenue par Jean-Baptiste Boisot. La quête d'un bénéfice la motivait, ainsi que le désir de contenter un protecteur et intercesseur grâce auquel maints présents d'érudition pouvaient parvenir aux pieds du trône. Le Bisontin était désireux de satisfaire cet ami par des savoirs qui pourraient enrichir ses travaux, mais ceux-ci furent souvent reçus dans l'indifférence et peut-être même avec une aimable condescendance, le courtisan étant affairé à d'autres tâches. Si Boisot prit l'initiative de cette circulation d'objets culturels et de savoirs, Pellisson l'orienta, la valorisa ou au contraire l'empêcha. Cette relation savante était le reflet, entre les deux hommes, de statuts sociaux et d'une reconnaissance au sein de la République des doctes disproportionnés. L'autorité qu'exerçait Pellisson sur son protégé ne fit que s'accentuer lorsqu'en 1687 il convia le Bisontin à collaborer à ses travaux. 


\section{Le collaborateur de Pellisson à la controverse religieuse}

\subsection{Le traducteur}

Boisot ne devait être à l'origine pour l'économe royal qu'un ami qui saurait l'informer dans les affaires spirituelles du pays conquis, ainsi qu'un agent de sa politique de reconquête religieuse. Le problème le plus urgent qui s'était posé à l'administrateur des économats avait été la diffusion de textes sacrés auprès des protestants à convertir et des nouveaux convertis. Dans l'été 1683, il convia son correspondant bisontin à distribuer une cinquantaine de ces ouvrages qu'il lui fit adresser par ses commis ou secrétaires. L'envoi comprenait notamment 30 exemplaires des Courtes prières durant la Sainte Messe, un opuscule que Pellisson avait composé pour lui-même après sa conversion et qui avait été publié en 1677 (Pellisson, Lettres inédites: 54-55). En désignant les institutions et les personnes à qui il fallait distribuer ces livres, le "grand convertisseur" ne laissait guère à son correspondant qu'un rôle d'exécutant. Il révéla un tempérament tout aussi directif lorsque s'instaurèrent entre les deux hommes les premières formes d'une collaboration érudite. Celle-ci se forma peu après que Pellisson eut commencé à placer sa plume au service de la controverse religieuse.

En 1686, il fit paraître son premier volume des Réflexions sur les différends de la religion (1686). L'ouvrage fut réimprimé l'année suivante, avec l'addition d'un nouveau tome intitulé Réponse aux objections d'Angleterre et de Hollande (1687). L'achevé d'imprimer date du 19 août 1687. Boisot reçut les deux livres à l'automne, qui n'étaient pas tant un don d'auteur que des instruments de travail accompagnant son invite à une collaboration. Avec Descartes, qui la préconisait pour le domaine scientifique dans son Discours de la méthode, les savants bénédictins du XVII ${ }^{\mathrm{e}}$ siècle avaient compris l'utilité d'une collaboration pour les travaux d'érudition monastique et se soumettaient régulièrement à sa discipline. Dispersé, comme commensal du roi, à maintes activités, mais désireux de compenser son manque de disponibilité par une efficacité dans ses tâches, Pellisson appréciait les avantages d'une œuvre partagée avec des collaborateurs. Il méditait, comme la grande affaire de sa vie, un Traité de l'Eucharistie qu'il voulait faire suivre d'un autre sur la Tradition, dont il espérait qu'ils mettraient un terme aux variations des Églises protestantes. Pour mener à bien cette œuvre, il aurait besoin d'un nombre important de traductions qu'il confiait à des personnes choisies. Dans une lettre datée du 19 novembre 1687, il proposa à l'abbé Boisot d'être du nombre des «ouvriers pour cette grande moisson» (Pellisson, Lettres inédites: 104).

Jusqu'en juillet 1689, le Bisontin joignit régulièrement à ses missives les traductions du traité de saint Jérôme Contre Vigilantius puis contre Jovinien, du De corona militis de Tertullien, ainsi que celle d'un traité de Lactance. Mais dans sa lettre datée du 26 novembre 1689, Pellisson lui confia qu'il n'était plus en mesure de lui prescrire de nouvelles tâches, le docte abbé ayant exécuté avec efficacité et zèle celles qui lui avaient été prescrites:

Je suis dans le Traité de l'Eucharistie que je reprends et pour lequel mes traductions sont faites ou prêtes il y a longtemps, et vous en avez vu une partie considérable déjà imprimée. Ce sera dans le Traité des Traditions où il y aura beaucoup de choses à traduire, mais encore qu'on y pût travailler dès cette heure, je crois qu'il vaut mieux attendre que je puisse m’appliquer moi-même à choisir ce qu'il faut prendre ou laisser et, cela, je ne le saurai proprement qu'en composant le traité général et les sections de chaque matière. (Pellisson, Lettres inédites: 123-124)

Cette collaboration savante, qui se traduisait par la circulation régulière, par voie épistolaire, de traductions des écrits des Pères de l'Église, était assujettie au rythme que lui donnait Pellisson. 


\subsection{Le découvreur de sources rares}

Boisot parvint néanmoins à s'extraire de cette dépendance intellectuelle en prenant l'initiative de rechercher dans ses archives des pièces rares qui pourraient avoir quelque utilité dans le débat avec les protestants. Il adressa à son ami, le premier de l'an 1692, la copie qui avait été envoyée au cardinal de Granvelle d'un bref circulaire du pape Pie IV du 6 avril 1564. L'acte, qui autorisait les évêques d'Allemagne à donner la communion sous les deux espèces (Mémoires, ms Granvelle 13: fo 127), était devenu introuvable du fait de sa révocation ultérieure par Rome. "Obligez-moi de me mander ce que vous en savez et le plus tôt que vous pourrez, car il ne serait pas impossible que je n'en fisse quelque usage dans cette nouvelle édition qui va finir», s'empressa de lui répondre Pellisson, à qui son ami venait de procurer une pièce essentielle pour convaincre d'un rapprochement possible des Églises (Pellisson, Lettres inédites: 175). Boisot joignit à sa missive datée du 20 janvier la lettre en italien qui accompagnait l'envoi au cardinal de Granvelle du bref pontifical. Écrite par le financier augsbourgeois Johann Jacob Fugger (1516-1575), il y était question du mariage des prêtres, que l'empereur Ferdinand I ${ }^{\text {er }}$ et son fils Maximilien $\mathrm{I}^{\mathrm{er}}$ réclamaient pour leurs États (Pellisson, Lettres inédites: 177; Additions: 94-95). Ce document apportait une autre preuve historique d'un compromis envisageable entre les Églises.

"Je vous remercie de la lettre italienne, je crois que je la ferai imprimer dans mes additions avec le bref latin et français et quelques extraits de vos lettres qui me parlent de cette matière ", promit Pellisson (Lettres inédites: 177). Ces sources parurent en effet en 1692 dans les additions de la quatrième partie de l'œuvre du controversiste, laquelle avait été exécutée par la nécessité de répondre aux contradictions du philosophe Leibniz (1646-1716) (Pellisson, Additions: 77-93; 96-102). Pellisson y livra également l'extrait d'une lettre recomposée adressée à l'abbé de Saint-Vincent, dans laquelle il flattait ce «FrancComtois qui vient disputer la politesse et la pureté à toute l'Académie française» et le remerciait de l'envoi de la copie du bref pontifical (Pellisson, Additions: 69-72). Il y joignit trois extraits de lettres que Boisot lui avait adressées (Pellisson, Additions: 69-76; 94-95).

Dans l'été 1692, l'abbé de Saint-Vincent confia à son ami un bref du pape Jules III au nonce de l'empereur touchant la conversion des princes protestants (Mémoires, ms Granvelle 4: fo 132), qu'il lui suggérait de communiquer à Leibniz (Pellisson, Lettres inédites: 181). La mort de Pellisson, le 7 février 1693, ne lui donna pas le temps d'en faire profit.

Alors qu'il n'avait été convié qu'à une collaboration accessoire à ce projet, comme traducteur de sources patristiques, laquelle était assujettie aux besoins du controversiste et se pliait à son rythme de travail, Boisot parvint à l'enrichir en exhumant de sa collection des documents essentiels. Pellisson ne se borna pas à les publier, mais il rendit dans son ouvrage un hommage appuyé à son collaborateur, lui procurant par la même occasion une reconnaissance auprès des doctes. L'abbé de Saint-Vincent s'attacha de son côté à concevoir un projet érudit dont il livra à son protecteur et ami certains matériaux, puis finalement l'ouvrage lui-même, espérant que celui-ci s'en ferait le promoteur chez les savants.

\section{L'héritier des papiers des Granvelle et son promoteur}

\subsection{Genèse d'une Vie de Granvelle et circulation de l'œuvre}

Jean-Baptiste Boisot confiait dans une lettre datée du 25 octobre 1686 à son ami Claude Nicaise, chanoine de la Sainte-Chapelle de Dijon, son projet d'honorer la mémoire des «beaux et bons esprits» de sa province: 
Depuis plus de deux cents ans, nous avons toujours eu des Premiers ministres dans quelques cours et des gens habiles qui ont écrit. [...] Je recherche avec soin tout ce qui reste de ces grands hommes. J'ai déjà recouvré un journal d'une diète de Francfort écrit par Monsieur de Brun, et une relation des négociations de la [diète] de Munster. [...] L'on pourrait quelque jour, si l'on était bien de loisir, donner au public la vie de quelques-uns de ces messieurs. (Correspondance de l'abbé Nicaise, ms Fr 9361, fo 51-52).

Le docte bisontin mûrissait probablement ce projet depuis qu'il avait commencé à sauver et collectionner les sources nécessaires à sa réalisation. Ce fut dans la décennie 1660 qu'il acheta au comte de La Baume-Saint-Amour, héritier des Granvelle, ainsi qu'au baron de Thoraise, les papiers du Cardinal (1517-1586) et de son père Nicolas Perrenot de Granvelle (1486-1550). Dans le même temps, le savant Jules Chifflet, abbé de Balerne (Jura) se consacrait également à la sauvegarde des documents de ce lignage, dont certains servaient alors d'emballage aux épiciers bisontins. Après la mort de cet érudit en 1676, Boisot acheta sa collection au parlementaire Philippe-Eugène Chifflet, frère du disparu ${ }^{3}$.

Plus réservé dans ses confidences à Pellisson sur ses projets qu'il ne l'était avec son ami dijonnais, l'abbé de Saint-Vincent ne paraît pas lui en avoir fait état avant l'envoi, le 6 juillet 1688, d'un court éloge de Jean de Saint-Mauris $\left(† 23\right.$ août $1555^{4}$ ). Ce personnage était le beau-frère du chancelier Nicolas Perrenot de Granvelle, auquel il devait son élévation. Après avoir débuté sa carrière comme conseiller au parlement de Dole, puis été conseiller et maître des requêtes ordinaires au Conseil privé des Pays-Bas en 1541, Jean de Saint-Mauris fut diplomate à la cour de France d'août 1541 à 1548, au service de la gouvernante des Pays-Bas puis comme ambassadeur impérial. Cet opuscule était probablement l'aboutissement du long travail de classement des archives, réunies en un volume (Lettres et papiers, ms Granvelle 70), de cette mission diplomatique qui devait clore les difficultés survenues dans l'exécution du traité de Crépy. Dans sa lettre de réponse, Pellisson se montre favorable au projet de Boisot et l'encourage à continuer:

J'ai vu avec plaisir votre petit éloge de Jean de Saint-Mauris et je comprends bien qu'un recueil de ces sortes de choses que vous déterrez dans vos manuscrits pourra être utile et faire honneur à votre patrie; ainsi je crois que vous ferez bien d'y employer le temps que vous aurez de reste. (Pellisson, Lettres inédites: 111)

Embarrassé dans sa controverse avec le pasteur calviniste Pierre Jurieu (1637-1713), très pris par sa charge de maître des requêtes ordinaires de l'Hôtel du roi qui le contraignait à siéger par quartiers trimestriels au Conseil des parties et à être de temps en temps rapporteur des affaires qui s'y jugeaient, Pellisson n'était plus en mesure de consacrer du temps à ses projets d'ouvrages auxquels Boisot apportait régulièrement ses traductions. Il choisit de libérer partiellement son collaborateur de cette activité, l'encourageant à se consacrer à son projet, ce qui n'était pas sans signification, puisque les personnalités qui intéressaient l'abbé de Saint-Vincent avaient servi les Habsbourg avec exemplarité. Leur carrière était évocatrice du destin brillant de la Comté et de ses élites sous leur domination. Loin de modérer la témérité de son ami, Pellisson lui réitéra bientôt ses encouragements: «[V]ous voyez que je puis vous donner un peu de répit en faveur de votre patrie» (26 novembre 1689). «Je suis fort aise d'apprendre

3. Boisot a relaté les étapes de l'acquisition de sa collection dans la préface de sa vie de Granvelle (Boisot, Lettre, ms 1244: fo 4).

4. Voir la «Notice de messire Jean de Saint-Mauris», écrite de la main de Boisot, conservée dans le recueil Copie des lettres et autres pièces concernant Mre Jean de St-Mauris (ms Fr 7122: 3-5). 
que vous continuez votre travail pour votre patrie à qui je ne doute pas qu'il ne fasse honneur quelque jour» (3 février 1690) (Pellisson, Lettres inédites: 124).

L'abbé de Saint-Vincent était non seulement assuré d'un protecteur et d'un conseiller dans l'élaboration de cette œuvre délicate, susceptible de faire douter de sa francophilie, mais il pouvait également en espérer une diffusion dans la République des Lettres grâce au soutien de l'une de ses plus illustres personnalités. Il fut pour la première fois question de cette Lettre ou Vie de Granvelle, qui était en réalité un inventaire sommaire et curieux des manuscrits de sa collection, dans une missive de Pellisson au Bisontin du 13 novembre 1692 (Pellisson: 187-188). Le courtisan lui recommandait d'exécuter un ouvrage consistant, «qui puisse résister au temps». «Hâtez-vous de me faire ce bien et ce plaisir pendant que je suis en état de le recevoir», lui demandait-il prophétiquement.

En janvier 1693, le précieux manuscrit devait être confié en main propre à Pellisson par le marquis de Renty (Pellisson, Lettres inédites: 192), lorsque la mort du courtisan interrompit sa circulation. Il fut renvoyé au Bisontin (Scudéry, Lettres: 113), qui l'adressa au printemps à Madeleine de Scudéry, l'amie de cœur du disparu. "Je vous exhorte, Monsieur, à continuer votre dessein et de trouver lieu de placer cette belle lettre qui fera honneur à l'illustre mort et à vous. Et je ne doute pas non plus que ce que vous écrivez n'en fasse beaucoup au cardinal de Granvelle» (Scudéry, Lettres: 114). Dans une missive plus tardive du 12 décembre, la «reine de Tendre» lui promettait de la lire

à ceux qui m'[en] ont fait la prière et je ne vous demande plus rien à cet égard, mais je vous demande toujours ce que Monsieur de Pellisson vous demandait, c'est-à-dire que ce que j’ai vu et que vous dites avoir retouché ne soit pas perdu, voie bientôt le jour pour votre gloire et celle de l'illustre mort. (Scudéry, Lettres: 136)

Mais privé de son protecteur, l'abbé de Saint-Vincent n'eut pas le temps de publier son œuvre, qui ne devait être livrée au public qu'en 1727.

\subsection{Circulation des sources}

L'ouvrage qu'il projetait se plaçait dans la continuité d'un fastidieux travail de tri et de classement, mais aussi de déchiffrement de documents diplomatiques cryptés. Plusieurs années furent nécessaires à l'accomplissement de cette tâche menée dans l'environnement studieux de l'abbaye de Saint-Vincent, redonnée aux études bénédictines par la congrégation vanniste, qui l'avait réformée en 1611. Le 8 novembre 1688, Boisot annonçait à son correspondant avoir décodé "cinq cents pages d'écriture " (Pellisson, Lettres inédites: 116). Le 10 juillet 1690, il lui faisait part de l'achèvement de ce classement, toutes ces sources ayant été classées en quatre-vingts volumes in-folio (Pellisson, Lettres inédites: 137). Cette tâche fastidieuse n'était pas tant menée pour lui permettre d'entreprendre un travail d'historien que pour faciliter l'accès du fonds aux érudits. Boisot s'empressait d'ailleurs d'ouvrir ses trésors à ceux de passage, comme en juillet 1683 à Mabillon et Michel Germain (Mabillon, Veterum analectorum : 6-7), ou à leur confrère mauriste Bernard de Montfaucon (Montfaucon, L'Antiquité expliquée : xxi-xxii). Pour d'autres, il se faisait dispensateur de documents transcrits de sa propre main, comme l'évêque de Nîmes et célèbre prédicateur Fléchier (Fléchier, Histoire : vi-vii), ou encore l'imprimeur-libraire Frédéric Léonard $\left(1624-1711^{5}\right)$.

5. «Lorsque Léonard imprima les derniers traités de paix, je lui fis offrir ceux que j'ai, dans la simple vue de lui faire plaisir. Mais son impression était trop avancée, ou il ne comprit pas que ce que je lui offrais était ce qu'il y 
Le savant collectionneur n'attendait-il pas de Pellisson qu'il se fasse son intermédiaire dans la diffusion de ces sources, ou tout au moins qu'il en révèle l'intérêt auprès de ses amis? N'était-ce pas dans cette attente que le Bisontin lui en procura la quintessence avec l'envoi de copies et, plus rarement, d'originaux? Or, faute de disponibilité, Pellisson ne sut pas apprécier la valeur de la copie d'une lettre de François $\mathrm{I}^{\mathrm{er}}$ à Charles Quint qui lui avait été adressée à l'automne 1685 (Pellisson, Lettres inédites: $\left.78^{6}\right)$. Il ne fut guère plus intéressé par celle des lettres de l'humaniste et philologue Henry Dupuys (1574-1646), ou par celle de missives de l'empereur Charles Quint et par deux autres du cardinal de Granvelle que son correspondant lui envoya le 7 juillet 1687 et dans l'été 1692 (Pellisson, Lettres inédites: 99, 181). Ces documents furent enfouis dans les "papiers historiques» du courtisan (Pellisson, Lettres inédites: 100).

La transcription d'apostilles de la main de Philippe II d'Espagne, retrouvées sur le courrier du monarque à ses administrateurs, fut en revanche communiquée dans l'été 1687 à Madeleine de Scudéry pour satisfaire sa curiosité et son goût de la langue espagnole (Pellisson, Lettres inédites: 97).

«Deux ou trois pièces très curieuses» signées par Marguerite d'Autriche ${ }^{7}$ furent réceptionnées à l'automne 1690 non plus comme des objets de curiosité, mais avec un savant intérêt. Il s'agissait d'originaux dont Boisot possédait le duplicata. Conscient de leur valeur, Pellisson indiqua vouloir les confier à quelque érudit susceptible d'en faire bon usage, ou bien à Monsieur Thévenot, bibliothécaire du roi (1620-1692) (Pellisson, Lettres inédites: 142). Il fut également très intéressé par l'Apologie d’Eunome, évêque de Cyzique († 395), dont Boisot possédait le manuscrit. L'on ne connaissait pas d'impression de cette œuvre, très utile à la compréhension des controverses trinitaires. La Nouvelle bibliothèque des auteurs ecclésiastiques devait même signaler en 1693 que l'«Apologie contre le traité de saint Basile» était perdue (Du Pin, Nouvelle bibliothèque: 387). Pellisson parla du manuscrit aux mauristes de l'abbaye de Saint-Germain-des-Prés, qui travaillaient justement à l'édition des Pères grecs. L'on s'accorda sur un prêt du document pour que les religieux puissent en réaliser une copie. Celui-ci fut remis au début de l'été 1691 dans les mains des mauristes et rendu à Pellisson à l'automne suivant (Pellisson, Lettres inédites: 145-146, 159-160, 169).

La diffusion des trésors déterrés par Boisot par la bienveillance de son protecteur n'était pas une garantie pour que ceux-ci soient durablement utiles à la communauté savante. En lui présentant ses vœux pour l'année 1691, l'abbé confiait à son ami son projet d'établir une bibliothèque publique (Pellisson, Lettres inédites: 147), dont il avait probablement approché le modèle dans le séjour qu'il fit en Italie au début de la décennie $1660^{\circ}$. Quelques jours avant sa mort, «pour l'avantage des gens doctes», le Bisontin légua le 27 novembre 1694 sa collection aux religieux de Saint-Vincent, à la condition qu'elle serait accessible deux fois par semaine à tous ceux qui désireraient la consulter?

aurait de plus curieux dans son recueil et il se contenta de me remercier», écrivait Boisot au chanoine Nicaise le 14 septembre 1694 (Correspondance de l'abbé Nicaise, ms Fr 9361: fo 75 vº).

6. Deux missives du Valois au Habsbourg sont conservées dans le fonds Granvelle, dont la Bibliothèque de Besançon est l'héritière. La première a pour objet la captivité du roi à Madrid (Mémoires, ms Granvelle 1: fo 82), la seconde est du 22 juin 1531 (Mémoires, ms Granvelle 2: fo 37).

7. Il n'est guère possible de préciser l'identité de la signataire. S'agissait-il de la duchesse douairière de Savoie (14801530), devenue gouvernante des Pays-Bas en 1506, ou bien de cette infante plus communément appelée Marie de Hongrie ou Marie d'Autriche (1505-1558), qui succéda à sa tante comme gouvernante des Pays-Bas? Ou bien de Marguerite de Parme ou d'Autriche (1522-1586), elle aussi gouvernante de cet héritage bourguignon en 1559?

8. Ce fut l'Église post-tridentine qui encouragea dans la Péninsule l'ouverture de bibliothèques publiques, à l'image de l'Ambrosiana de Milan ou de l'Angelica de Rome.

9. Voir l'Inventaire des manuscrits, livres, médailles, peintures... (ms 1268). 
Jean-Baptiste Boisot réussit donc à s'émanciper de la collaboration scientifique passive à laquelle l'avait convié son protecteur, en concevant un projet d'érudition aussi risqué qu'original, nourri de «choses nouvelles et singulières» (Boisot, Lettre, ms 1244: $\mathrm{f}^{\circ} 2 \mathrm{r}^{\circ}$ ). Mais le Bisontin n'aurait su parvenir à une reconnaissance dans la République des doctes sans cette protection qui n'avait rien d'un éteignoir: ce fut Pellisson qui l'encouragea à mener son œuvre à bien et qui était, par ses relations, en mesure de la soumettre à une large diffusion. Il eut également un rôle essentiel dans la diffusion des archives des Granvelle.

Durant les vingt années de la correspondance qui nous en est conservée, Paul Pellisson-Fontanier et Jean-Baptiste Boisot se transmirent maints savoirs et objets de connaissance qui ne furent pas seulement utiles à leur œuvre d'érudition, mais qu'ils surent mettre à la disposition des doctes en en favorisant la diffusion. Cette circulation savante fut constamment en évolution du fait des transformations qui s'opérèrent dans leurs rapports et dans leurs projets.

Leur commerce reposait sur une foncière inégalité de puissance, d'un point de vue aussi bien politique que scientifique, laquelle était susceptible de maintenir Jean-Baptiste Boisot dans une fonction exiguë de collaborateur, notamment lorsque «le plus grand convertisseur du royaume» (Puaux 1861: 38) le convia en 1687 à l'aider dans son œuvre de controversiste. Mais Boisot sut intelligemment tirer parti de cette collaboration pour faire apprécier son érudition et donner aux savants le désir d'exploiter son fonds d'archives.

En janvier 1693, Leibniz chercha à se procurer le catalogue de ces richesses par l'entremise du chanoine Nicaise. Boisot répondit à ce dernier dans une lettre du 8 décembre 1693: "Monsieur de Leibniz vous parle trop magnifiquement de mon pauvre petit trésor. Je fouillerai dedans l'un de ces jours, et je verrai ce qui pourra être de sa convenance. Le mal est qu'il faut copier exactement, que peu de gens en sont capables et que je n'ai pas grand loisir" (Correspondance de l'abbé Nicaise, ms Fr 9361: fo $59 \mathrm{r}^{\circ}$ ). Cette réponse est révélatrice de la réputation que l'abbé Boisot venait d'acquérir parmi les savants de son temps, la notoriété et les bonnes grâces de Pellisson n’y étant pas étrangères.

\section{Références bibliographiques}

Boisot, J.-B. 1692. Lettre de l'abbé J.-B. Boisot à Pellisson, sur un projet de biographie du cardinal de Granvelle. Ms 1244. Bibliothèque municipale d'étude et de conservation: Besançon.

Boisot, J.-B. 1727. «Lettre de M. Jean-Baptiste Boisot [...] à M. Pellisson [...] contenant un projet de la vie du cardinal de Granvelle, qu'il avait dessein d'écrire». Continuation des mémoires de littérature et d'bistoire. 4 (I): 26-167.

Bosquillon, N. 1695. «Lettre de Monsieur Bosquillon à Mademoiselle de Scudéry, contenant l'éloge de Monsieur l'Abbé Boisot». Journal des sçavans, pour l'année M. DC. XCV (Amsterdam) 23 (6 juin) : 383-397.

Copie des lettres et autres pièces concernant Mre Jean de St-Mauris, ambassadeur de Charles V auprès de François Ier,... recueillis... par M. Boisot, abbé de S. Vincent de Besançon. 1544-1554. Ms Français 7122. Bibliothèque nationale de France: Paris.

Correspondance de l'abbé Nicaise, chanoine de la Ste-Chapelle de Dijon. 1660-1701. Ms Français 9361. Bibliothèque nationale de France: Paris.

Du Pin, L.-E. 1693. Nouvelle bibliothèque des auteurs ecclésiastiques, vol. 2. Paris: A. Pralard.

Fléchier, E. 1693. Histoire du cardinal Ximenès. Paris : J. Anisson. 
Goncourt, J. et E. de. 1903. Portraits intimes du XVIII siècle: Études nouvelles d'après les lettres autographes et les documents inédits. Paris: E. Fasquelle. [1857].

Inventaire des manuscrits, livres, médailles, peintures, pieds d'estaux, donnés par Monsieur l'abbé Boizot pour une bibliothèque publique à Besançon. 1694. 1695. Ms 1268. Bibliothèque municipale d'étude et de conservation: Besançon.

Isaac, M.-T. et C. Sorgeloos, éd. 1996. La diffusion du savoir scientifique XVI-XIX siècles : actes du colloque de l'Université de Mons-Hainaut, 22 septembre 1995. Bruxelles: Archives et Bibliothèques de Belgique.

Lettres et papiers de l'ambassade de Jean de Sainct Mauris en France. 1544-1582. Ms Granvelle 70. Bibliothèque municipale d'étude et de conservation: Besançon.

Mabillon, J. 1685. Veterum analectorum tomus IV. Paris: Vve E. Martin et J. Boudot.

Marcou, F. L. 1859. Étude sur la vie et les œuvres de Pellisson, suivie d'une correspondance inédite du même. Paris: Didier.

Marchal, R. 1996. «Deux doctes entre Dieu et le roi: Les lettres inédites de Paul Pellisson à l’abbé Jean-Baptiste Boisot». Dans Aspects du classicisme et de la spiritualité: Mélanges en l'honneur de Jacques Hennequin. Paris: Klincksieck : 339-352.

Martène, E. et U. Durand. 1717. Voyage littéraire de deux religieux bénédictins de la congrégation de Saint-Maur, 2 parties en 1 vol. Paris: F. Delaulne.

Mauriès, P. 2011. Cabinets de curiosités. Paris: Gallimard. [2002].

Mémoires de ce qui sest passé sous le ministère du chancelier et du cardinal de Granvelle, ramassées [sic] par messire Jean-Baptiste Boisot, abbé de Sainct-Vincent de Besançon. Ms Granvelle 1 (1408-1529), 2 (1530-1536), 4 (1546-1607), 13 (juillet-août 1564). Bibliothèque municipale d'étude et de conservation: Besançon.

Montfaucon, B. de. 1719. L'Antiquité expliquée et représentée en figures, vol. 1. Paris: F. Delaulne.

Pellisson-Fontanier, P. 1674-1693. Lettres de M. Pellisson-Fontanier... à M. Boisot, abbé de Saint-Vincent [de Besançon]. Ms. 602. Bibliothèque municipale d'étude et de conservation: Besançon.

Pellisson-Fontanier, P. 1686. Réflexions sur les différends de la religion, avec les preuves de la tradition ecclésiastique. Paris: G. Martin.

Pellisson-Fontanier, P. 1687. Réflexions sur les différends de la religion, seconde partie: Réponse aux objections d'Angleterre et de Hollande, ou de l'autorité du grand nombre dans la religion chrétienne. Paris: G. Martin.

Pellisson-Fontanier, P. 1692. Additions. Dans De la tolérance des religions. Lettres de M. de Leibniz et réponses de M. Pellisson. Paris: J. Anisson: 1-185 (pagination séparée).

Pellisson-Fontanier, P. 1729. Histoire de la dernière guerre entre la France \& l'Espagne, ou de la conquête de la FrancheComté. Dans Continuation des mémoires de littérature et d'histoire. 7 (I) : 3-199.

Pellisson-Fontanier P. 2016. Lettres inédites à l'abbé Jean-Baptiste Boisot (1674-1693). Édition préparée par C. Marchal et F. Marchal-Ninosque. Paris: Classiques Garnier.

Puaux, F. 1861. Histoire de la réformation française, vol. 6. Paris: Lévy frères.

Registres paroissiaux. Paroisse Saint-Pierre: Baptêmes. 1636-1662. GG 177. Archives municipales: Besançon.

Ribier, G. 1666. Lettres et mémoires d'Estat des roys, princes, ambassadeurs et autres ministres, sous les règnes de François premier, Henry II et François II, 2 vol. Paris: F. Clouzier.

Saule, B. et C. Arminjon. 2010, dir. Sciences et curiosités à la cour de Versailles. Paris: Réunion des Musées Nationaux.

Schnapper, A. 2012. Le géant, la licorne et la tulipe: Les cabinets de curiosités en France au XVII siècle. Histoire et histoire naturelle. Édition préparée par S. Mouquin, M. Patrick et S. Mickaël. Paris: Flammarion. [1988].

Scudéry, M. de. 2019. Lettres à l'abbé Jean-Baptiste Boisot et à Jeanne-Anne de Bordey-Chandiot (1686-1699). Édition préparée par C. Marchal. Paris: Classiques Garnier.

Veillon, M. 1997. «La science des médailles antiques sous le règne de Louis XIV». Revue numismatique. 152: 359-377. 